\title{
Pedometer-determined physical activity among youth in the Tokyo Metropolitan area: a cross-sectional study
}

\author{
Noritoshi Fukushima', Shigeru Inoue ${ }^{1 *}$, Yuki Hikihara² ${ }^{2}$ Hiroyuki Kikuchi ${ }^{1}$, Hiroki Sato ${ }^{1}$, Catrine Tudor-Locke ${ }^{3}$ \\ and Shigeho Tanaka ${ }^{4}$
}

\begin{abstract}
Background: Providing large-scale descriptive data of objectively measured physical activity in youth is informative for practitioners, epidemiologists, and researchers. The purpose of this study was to present the pedometer-determined physical activity among Japanese youth using the Tokyo Metropolitan Survey of Physical Fitness, Physical Activity and Lifestyle 2011.

Methods: This study used a school-based survey. The Tokyo Metropolitan Board of Education originally collected pedometer-determined steps per day in the fall of 2011. Data were collected from 15,471 youth aged 6 to 18 years living in Tokyo. Participants were asked to wear pedometers for 14 consecutive days, and daily steps logged in the final 7 days were selected for this analysis.

Results: At the primary and junior high school levels, boys (12,483 and 9476, respectively) had a significantly higher mean number of steps per day than did girls (10,053 and 8408, respectively). There was no significant difference in the mean number of steps per day between the sexes at the high school level. Mean steps per day decreased consistently with age and grade level; the lowest overall steps per day was observed in the last year of junior high school, although there was a slight increase in the subsequent year, the first year of high school.

Conclusions: This study demonstrates a trend toward reduced physical activity with age in Japanese youth and a substantial difference in the number of steps per day between boys and girls in Tokyo. The age-related reduction in steps per day was greater in boys because they attained a higher peak value prior to this reduction, and sex-related differences in the step count disappeared in high school students.
\end{abstract}

Keywords: Survey, Steps, Children, Adolescents, Cross-sectional study, Descriptive epidemiology

\section{Background}

Lack of physical activity (PA) in childhood and adolescence is associated with adverse health problems such as obesity and increased cardiovascular and diabetes risk $[1,2]$. Childhood PA patterns often extend into adulthood; insufficient PA during this developmental period is therefore a great public health threat [3]. To improve health outcomes, the World Health Organization recommends that children and adolescents (hereafter collectively termed "youth") aged 5 to 17 years participate in a

\footnotetext{
* Correspondence: inoue@tokyo-med.ac.jp

1 Department of Preventive Medicine and Public Health, Tokyo Medical

University, 6-1-1 Shinjuku Shinjuku-ku, Tokyo 160-8402, Japan

Full list of author information is available at the end of the article
}

daily minimum of 60 min of moderate to vigorous PA [4]. Despite these recommendations, physical activity levels among youth remain low worldwide $[5,6]$.

Previous studies that evaluated PA levels primarily used standardized self-report questionnaires $[2,5,6]$. Although self-reporting is reasonable for large-scale epidemiological investigations, it may be less appropriate for measuring PA in children and adolescents. For example, recall bias may affect the accuracy of child data more than adult data [7]. Additionally, children may be unable to accurately summarize the sporadic and complex nature of their PA when responding to questions about habitual behavior [8, 9]. Further, cross-national comparisons of self-reported PA are affected by language 
and cultural differences [10-12]. Collectively, these concerns indicate a substantial need for objectively measured youth PA [13].

Pedometers and accelerometers are commonly used to objectively measure PA and are increasingly used as research tools. It has also been reported that these devices are valid and feasible in assessing PA in youth [14]. Moreover, pedometers are more cost-effective than accelerometers; the number of steps per day provides simple and practical information about PA volume for researchers, practitioners, and the lay public. There are a few examples of national surveys of young people's objectively determined steps per day: the Canadian Physical Activity Levels among Youth (CANPLAY) survey used pedometers $[15,16]$, the U.S. National Health and Nutrition Examination Survey used accelerometers [17], and the European Youth Heart Study also used accelerometers [18]. Still, evidence regarding objectively measured PA among Asian youth are quite limited [5].

We aimed to examine descriptive epidemiological data for children's and adolescents' pedometer-determined PA levels using the Tokyo Metropolitan Survey of Physical Fitness, Physical Activity and Lifestyle 2011. Specifically, we provide descriptive epidemiological data on the number of steps per day, stratified by sex and grade level.

\section{Methods}

A schematic depicting the sampling and data assessment methodology used in this study is shown in Fig. 1.

\section{Data source}

The Tokyo Metropolitan Board of Education (TMBE) performed a cross-sectional survey to investigate PA in youth living in Tokyo by examining the number of pedometer-recorded steps per day during the 2011 fall academic term. The TMBE authority and the Tokyo Metropolitan Government approved the secondary use of these data for research purposes, and all provided data were stripped of personal identifiers.

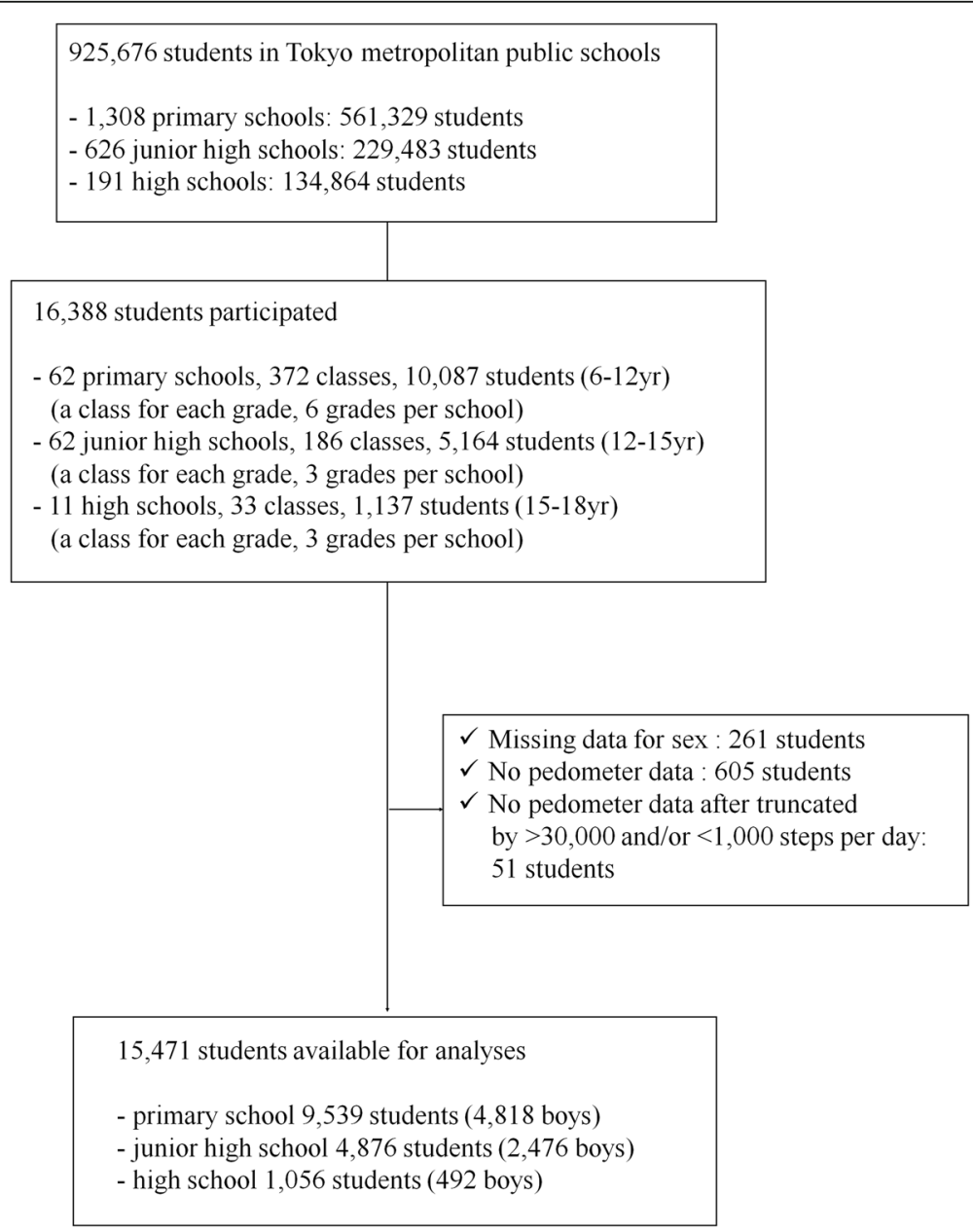

Fig. 1 Participant sampling flow chart and strategy for data assessment 


\section{Participants and data collection}

Primary school and junior high school are compulsory in Japan. Children are admitted to primary school at 6 years of age. They spend 6 years in primary school, followed by 3 years in junior high school. After graduation from public junior high school in 2011, $97.6 \%$ of students in Tokyo attended high school for 3 years [19]. In Tokyo in June 2011, there were 561,329 students registered in 1308 public primary schools, 229,483 students in 626 public junior high schools, and 134,864 students in 191 public high schools. Geographically, the Tokyo metropolitan comprises 2 areas and 2 islands containing 62 municipalities: $23 \mathrm{Ku}$-Area (23 wards), Tama-Area (26 cities, 3 towns, and 1 village), and Izu and Ogasawara Islands ( 2 towns and 7 villages). This is a secondary analysis of a TMBE survey that did not employ random sampling. Instead each of the 62 municipalities of Tokyo were asked, at their own discretion to designate one public primary school and one public junior high school from their jurisdiction for targeted measurement. Thus, the data came from 62 primary schools and 62 junior high schools throughout Tokyo. One class per grade in each of these schools participated in this survey. The TMBE sampled high school students from the 11 school districts of the Tokyo metropolitan.

Data were collected from 10,087 students from 62 primary schools (372 classes), 5164 students from 62 junior high schools (186 classes), and 1137 students from 11 public high schools (33 classes). Participants were aged 6 to 18 years. Each school held an orientation meeting for this survey for participants and their parents or guardians in August 2011. Data were collected using pedometers and questionnaires during the fall term of 2011 (September to November).

\section{Pedometer-determined PA}

TMBE members chose the pedometer used in the survey (EX-200; Yamasa Co., Ltd., Tokyo, Japan; approximately \$US 23); Yamasa is the Japanese generic name for Yamax, and this brand has been commonly used among PA researchers [20, 21]. In addition, our previous study reported acceptable comparability of the EX-200 compared with the SW-200 (Yamax Co., Ltd., Tokyo, Japan), the Kenz Lifecorder (Suzuken Corp., Nagoya, Japan), and the Active style Pro HJA-350IT (Omron Healthcare, Kyoto, Japan) among Japanese children [22]. Pedometers were placed in participants' pockets for data collection in the present study. The EX-200 can store up to 7 days of memory data, and students recorded their step counts daily using the memory function at school under the guidance of trained teachers. Participants were asked to wear an unsealed pedometer during waking hours for 14 consecutive days; they were allowed to remove the device for water-based activities and while engaging in full- contact sports (e.g., judo). The number of steps per day during the first 7 days of monitoring was not recorded in accordance with the original TMBE survey protocol. Therefore, the data for the remaining 7 days were used in these analyses.

\section{Data treatment and statistical analyses}

Step data were treated similarly to those of the CANPLAY survey [23] to enable comparison of both sets of results. Because a single day of pedometer data can be used to accurately estimate PA levels for surveillance purposes, participants aged 6 to 18 years with at least 1 valid day of pedometer data were included in this analysis [23, 24]. Records of $<1,000$ or $>30,000$ steps per day were considered outliers and excluded from further analyses [15, 20,23]. Valid days were thus defined as any day with recorded data between these two thresholds. Descriptive data (means, $95 \%$ confidence intervals [CI]) for the number of steps per day were calculated based on the number of valid days for each grade level and sex (combined and separately). Ranges and percentile values were calculated for each grade level by sex. In Japan, an evidence-based recommendation for steps per day for youth aged 6 to 18 years has not yet been established. Therefore, we used criteria applied in past studies to describe the proportion of participants taking $\geq 10,000$, $\geq 12,000$, and 15,000 steps per day $[15,25,26]$. Specifically, these criteria were a separate body mass indexreferenced criteria for boys and girls (15,000 and 12,000 steps/day, respectively) $[15,26]$ and a step count related to $60 \mathrm{~min}$ of moderate to vigorous PA for adolescent boys and girls (10,000 steps per day) [25]. Moreover, 15,000 steps per day is the target recommended by the Tokyo Metropolitan Government for boys and girls aged 6 to 18 years [27] and $<7000$ steps per day is a potential candidate for the lower threshold in children, which indicates a sedentary lifestyle [28]. Finally, an accumulated $<5000$ steps per day (originally considered to indicate a sedentary lifestyle for adults) was used as an alternative marker of a sedentary lifestyle [28]. Student's $t$-test was used to test for sex differences, stratified for each grade level. Cohen's $d$ effect size index was used to assess the magnitude of intergroup differences and statistical significance [29]. All statistical procedures and calculations of $p$-values were conducted using two-tailed t-tests. Differences were considered statistically significant at $p<0.05$. Statistical analyses were performed using IBM SPSS software, version 21.0 (IBM, Armonk, NY, USA).

\section{Results}

A total of 16,388 students participated in this survey. We excluded 261 students whose sex was unspecified in the data set, 605 students without any pedometer data recorded during the final 7 days of the monitoring 
period, and 51 students with no pedometer data after truncation to $<1000$ or $>30,000$ steps per day as data outliers. Thus, step-defined PA was successfully measured for 15,471 students, each with at least 1 valid day of data. Overall, $3.1 \%$ of boys and $1.6 \%$ of girls had only 1 valid day of pedometer data; $86.2 \%$ of boys and $90.7 \%$ of girls had $\geq 4$ valid days of pedometer data (Table 1).

Table 2 shows the mean number of steps per day and $95 \% \mathrm{CI}$, stratified by sex and grade level. The highest mean number of steps per day $(11,659)$ was found among first-grade students in primary school (6-7 years old) and consistently decreased with age. The lowest mean number of steps per day (7887) was observed in students in the last year of junior high school (1415 years old). Beyond junior high school, the mean step count modestly increased to 8485 steps per day in the first year of high school (15-16 years old), but declined as students advanced through high school (8032 steps per day at 17-18 years old). For boys, the mean number of steps per day increased from 12,575 in the first grade of primary school, peaked at 12,736 in the third grade of primary school, and subsequently ranged from 8337 to 10,218 in junior high school and from 7935 to 8583 in high school. For girls, the mean number of steps per day followed the same trend seen for both sexes combined: the highest mean number of steps per day was observed in the first grade of primary school $(10,694)$ and consistently declined through junior high school (7437-9104), with a slight increase in the minimum value of the range in high school (8025-8398).

During primary and junior high school, the step count was significantly higher among boys by 2000 and 1000

Table 1 Distribution of participants by valid days of pedometer wear, grade, and sex in Tokyo in 2011

\begin{tabular}{|c|c|c|c|c|c|c|c|c|c|c|c|c|c|c|c|c|c|c|}
\hline & \multirow[b]{3}{*}{ Grade } & \multirow[b]{3}{*}{ Age } & & & \multicolumn{14}{|c|}{ Number of students per valid days of pedometer wear } \\
\hline & & & \multicolumn{2}{|l|}{ Total } & \multicolumn{2}{|c|}{1 day } & \multicolumn{2}{|c|}{2 days } & \multicolumn{2}{|c|}{3 days } & \multicolumn{2}{|c|}{4 days } & \multicolumn{2}{|c|}{5 days } & \multicolumn{2}{|c|}{6 days } & \multicolumn{2}{|c|}{7 days } \\
\hline & & & $n$ & $\mathrm{Pct}$ & $n$ & Pct & $n$ & PCt & $n$ & Pct & $n$ & Pct & $n$ & Pct & $n$ & Pct & $n$ & Pct \\
\hline \multicolumn{19}{|l|}{ Boys } \\
\hline \multirow[t]{7}{*}{ Primary school } & All grades & & 7786 & 100 & 245 & 3.1 & 344 & 4.4 & 490 & 6.3 & 830 & 10.7 & 1220 & 15.7 & 1572 & 20.2 & 3085 & 39.6 \\
\hline & 1 & $6-7$ & 802 & 100 & 22 & 2.7 & 38 & 4.7 & 48 & 6.0 & 74 & 9.2 & 117 & 14.6 & 182 & 22.7 & 321 & 40.0 \\
\hline & 2 & $7-8$ & 772 & 100 & 30 & 3.9 & 37 & 4.8 & 55 & 7.1 & 76 & 9.8 & 115 & 14.9 & 179 & 23.2 & 280 & 36.3 \\
\hline & 3 & $8-9$ & 768 & 100 & 33 & 4.3 & 46 & 6.0 & 52 & 6.8 & 92 & 12.0 & 106 & 13.8 & 168 & 21.9 & 271 & 35.3 \\
\hline & 4 & $9-10$ & 828 & 100 & 33 & 4.0 & 33 & 4.0 & 47 & 5.7 & 84 & 10.1 & 132 & 15.9 & 173 & 20.9 & 326 & 39.4 \\
\hline & 5 & $10-11$ & 795 & 100 & 22 & 2.8 & 35 & 4.4 & 41 & 5.2 & 77 & 9.7 & 136 & 17.1 & 155 & 19.5 & 329 & 41.4 \\
\hline & 6 & $11-12$ & 853 & 100 & 27 & 3.2 & 29 & 3.4 & 45 & 5.3 & 77 & 9.0 & 123 & 14.4 & 181 & 21.2 & 371 & 43.5 \\
\hline \multirow[t]{3}{*}{ Junior high school } & 1 & $12-13$ & 877 & 100 & 34 & 3.9 & 40 & 4.6 & 70 & 8.0 & 119 & 13.6 & 151 & 17.2 & 174 & 19.8 & 289 & 33.0 \\
\hline & 2 & $13-14$ & 783 & 100 & 16 & 2.0 & 33 & 4.2 & 59 & 7.5 & 106 & 13.5 & 131 & 16.7 & 146 & 18.6 & 292 & 37.3 \\
\hline & 3 & $14-15$ & 816 & 100 & 23 & 2.8 & 38 & 4.7 & 52 & 6.4 & 79 & 9.7 & 128 & 15.7 & 138 & 16.9 & 358 & 43.9 \\
\hline \multirow[t]{3}{*}{ High school } & 1 & $15-16$ & 177 & 100 & 3 & 1.7 & 9 & 5.1 & 13 & 7.3 & 15 & 8.5 & 34 & 19.2 & 16 & 9.0 & 87 & 49.2 \\
\hline & 2 & $16-17$ & 161 & 100 & 0 & 0.0 & 4 & 2.5 & 3 & 1.9 & 21 & 13.0 & 21 & 13.0 & 30 & 18.5 & 82 & 50.9 \\
\hline & 3 & $17-18$ & 154 & 100 & 2 & 1.3 & 2 & 1.3 & 5 & 3.2 & 10 & 6.5 & 26 & 16.9 & 30 & 19.5 & 79 & 51.3 \\
\hline \multicolumn{19}{|l|}{ Girls } \\
\hline \multirow[t]{7}{*}{ Primary school } & All grades & & 7685 & 100 & 124 & 1.6 & 232 & 3.0 & 357 & 4.6 & 627 & 8.2 & 949 & 12.3 & 1575 & 20.5 & 3821 & 49.7 \\
\hline & 1 & $6-7$ & 762 & 100 & 18 & 2.4 & 26 & 3.4 & 47 & 6.2 & 78 & 10.2 & 88 & 11.5 & 160 & 21.0 & 345 & 45.3 \\
\hline & 2 & $7-8$ & 776 & 100 & 12 & 1.5 & 31 & 4.0 & 37 & 4.8 & 83 & 10.7 & 92 & 11.9 & 144 & 18.6 & 377 & 48.6 \\
\hline & 3 & $8-9$ & 797 & 100 & 18 & 2.3 & 33 & 4.1 & 36 & 4.5 & 60 & 7.5 & 88 & 11.0 & 181 & 22.7 & 381 & 47.8 \\
\hline & 4 & $9-10$ & 787 & 100 & 12 & 1.5 & 22 & 2.8 & 39 & 5.0 & 68 & 8.6 & 97 & 12.3 & 162 & 20.6 & 387 & 49.2 \\
\hline & 5 & $10-11$ & 796 & 100 & 11 & 1.4 & 20 & 2.5 & 22 & 2.8 & 47 & 5.9 & 76 & 9.5 & 166 & 20.9 & 454 & 57.0 \\
\hline & 6 & $11-12$ & 803 & 100 & 10 & 1.2 & 14 & 1.7 & 34 & 4.2 & 50 & 6.2 & 91 & 11.3 & 146 & 18.2 & 458 & 57.0 \\
\hline \multirow[t]{3}{*}{ Junior high school } & 1 & $12-13$ & 838 & 100 & 12 & 1.4 & 24 & 2.9 & 53 & 6.3 & 73 & 8.7 & 134 & 16.0 & 184 & 22.0 & 358 & 42.7 \\
\hline & 2 & $13-14$ & 746 & 100 & 11 & 1.5 & 16 & 2.1 & 36 & 4.8 & 70 & 9.4 & 81 & 10.9 & 158 & 21.2 & 374 & 50.1 \\
\hline & 3 & $14-15$ & 816 & 100 & 13 & 1.6 & 31 & 3.8 & 33 & 4.0 & 58 & 7.1 & 108 & 13.2 & 166 & 20.3 & 407 & 49.9 \\
\hline \multirow[t]{3}{*}{ High school } & 1 & $15-16$ & 197 & 100 & 5 & 2.5 & 7 & 3.6 & 8 & 4.1 & 20 & 10.2 & 33 & 16.8 & 39 & 19.8 & 85 & 43.1 \\
\hline & 2 & $16-17$ & 213 & 100 & 1 & 0.5 & 6 & 2.8 & 5 & 2.3 & 14 & 6.6 & 38 & 17.8 & 42 & 19.7 & 107 & 50.2 \\
\hline & 3 & $17-18$ & 154 & 100 & 1 & 0.6 & 2 & 1.3 & 7 & 4.5 & 6 & 3.9 & 23 & 14.9 & 27 & 17.5 & 88 & 57.1 \\
\hline
\end{tabular}


Table 2 Mean number of steps per day (with $95 \%$ Cl) among boys and girls by grade level in Tokyo in 2011

\begin{tabular}{|c|c|c|c|c|c|c|c|c|c|c|c|c|c|c|}
\hline & \multirow[b]{2}{*}{ Grade } & \multirow[b]{2}{*}{ Age } & \multicolumn{3}{|l|}{ Total } & \multicolumn{3}{|l|}{ Boys } & \multicolumn{3}{|l|}{ Girls } & \multirow{2}{*}{$\begin{array}{l}\text { Differences of steps } \\
\text { per day between } \\
\text { boys and girls }\end{array}$} & \multirow[b]{2}{*}{$p$ value } & \multirow[b]{2}{*}{$d$} \\
\hline & & & $n$ & $\begin{array}{l}\text { Mean steps } \\
\text { per day }\end{array}$ & $95 \% \mathrm{Cl}$ & $n$ & $\begin{array}{l}\text { Mean steps } \\
\text { per day }\end{array}$ & $95 \% \mathrm{Cl}$ & $n$ & $\begin{array}{l}\text { Mean steps } \\
\text { per day }\end{array}$ & $95 \% \mathrm{Cl}$ & & & \\
\hline All grades & & & 15471 & 10338 & $10281-10395$ & 7786 & 11262 & $11173-11351$ & 7685 & 9402 & $9336-9468$ & 1860 & $<0.001$ & 0.513 \\
\hline \multirow[t]{6}{*}{ Primary school } & 1 & $6-7$ & 1564 & 11659 & $11504-11813$ & 802 & 12575 & 12369-12781 & 762 & 10694 & 10482-10906 & 1881 & $<0.001$ & 0.602 \\
\hline & 2 & $7-8$ & 1548 & 11641 & $11475-11806$ & 772 & 12714 & $12491-12936$ & 776 & 10573 & 10352-10795 & 2141 & $<0.001$ & 0.644 \\
\hline & 3 & $8-9$ & 1565 & 11573 & $11406-11740$ & 768 & 12736 & $12512-12961$ & 797 & 10452 & $10232-10673$ & 2284 & $<0.001$ & 0.677 \\
\hline & 4 & $9-10$ & 1615 & 11336 & 11169-11503 & 828 & 12596 & $12381-12811$ & 787 & 10010 & 9790-10231 & 2586 & $<0.001$ & 0.758 \\
\hline & 5 & $10-11$ & 1591 & 10908 & 10740-11077 & 795 & 12302 & $12085-12519$ & 796 & 9517 & $9300-9734$ & 2785 & $<0.001$ & 0.815 \\
\hline & 6 & $11-12$ & 1656 & 10612 & 10444-10781 & 853 & 12021 & $11807-12234$ & 803 & 9117 & 8896-9337 & 2904 & $<0.001$ & 0.831 \\
\hline \multirow[t]{3}{*}{ Junior high school } & 1 & $12-13$ & 1715 & 9674 & $9497-9850$ & 877 & 10218 & 9974-10462 & 838 & 9104 & $8855-9353$ & 1114 & $<0.001$ & 0.299 \\
\hline & 2 & $13-14$ & 1529 & 9273 & $9092-9455$ & 783 & 9830 & 9580-10080 & 746 & 8689 & 8433-8946 & 1141 & $<0.001$ & 0.316 \\
\hline & 3 & $14-15$ & 1632 & 7887 & $7743-8031$ & 816 & 8337 & $8136-8538$ & 816 & 7437 & $7236-7638$ & 900 & $<0.001$ & 0.304 \\
\hline \multirow[t]{3}{*}{ High school } & 1 & $15-16$ & 374 & 8485 & $8147-8824$ & 177 & 8583 & $8091-9075$ & 197 & 8398 & $7932-8864$ & 185 & 0.592 & \\
\hline & 2 & $16-17$ & 374 & 8152 & $7828-8477$ & 161 & 8322 & $7827-8816$ & 213 & 8025 & $7595-8454$ & 297 & 0.373 & \\
\hline & 3 & $17-18$ & 308 & 8032 & $7692-8373$ & 154 & 7935 & $7452-8417$ & 154 & 8130 & $7647-8612$ & -195 & 0.574 & \\
\hline \multicolumn{15}{|l|}{ (subclassification) } \\
\hline \multirow[t]{3}{*}{ Primary school } & $1-3$ & $6-9$ & 4677 & 11624 & 11530-11718 & 2342 & 12674 & $12542-12806$ & 2335 & 10571 & 10439-10703 & 2103 & $<0.001$ & 0.642 \\
\hline & $4-6$ & $9-12$ & 4862 & 10950 & 10853-11047 & 2476 & 12303 & $12175-12432$ & 2386 & 9545 & $9414-9676$ & 2758 & $<0.001$ & 0.798 \\
\hline & $1-6$ & $6-12$ & 9539 & 11280 & $11212-11348$ & 4818 & 12483 & $12382-12584$ & 4721 & 10053 & 9977-10129 & 2430 & $<0.001$ & 0.718 \\
\hline Junior high school & $1-3$ & $12-15$ & 4876 & 8950 & $8851-9049$ & 2476 & 9476 & $9347-9604$ & 2400 & 8408 & $8278-8539$ & 1068 & $<0.001$ & 0.302 \\
\hline High school & $1-3$ & $15-18$ & 1056 & 8235 & $8042-8428$ & 492 & 8294 & $8007-8582$ & 564 & 8184 & $7915-8452$ & 110 & 0.575 & \\
\hline
\end{tabular}


steps per day, respectively, compared with girls at the same grade level. However, during the high school years, there was no significant sex difference in numbers of daily steps $(p=0.592$ for high school level 1 [15-16 years], $p=0.373$ for level 2 [16-17 years], and $p=0.574$ for level 3 [17-18 years]), with boys taking an absolute average of only 200 steps per day more than girls (Table 2). Table 3 shows the minimum, maximum, and percentile values for the number of steps per day in each school grade, stratified by sex.

Table 4 compares the results of this study, which used Yamasa EX-200 pedometers, and those of the CANPLAY survey, which used Yamax Digiwalker SW-200 pedometers [16]. The mean number of daily steps taken by boys in primary school was similar for the two surveys. However, at the higher-grade levels, pedometer-determined PA levels were lower for boys living in Tokyo than for their Canadian counterparts by approximately 1000 to 2000 steps per day. Similarly, the number of steps taken per day by girls living in Tokyo was lower by approximately 1000 steps per day than that of Canadian girls across all grades.

The proportion of students taking $\geq 10,000, \geq 12,000$, and $\geq 15,000$ steps per day was $60.5,41.2$, and $17.4 \%$, respectively, for boys and 39.1, 17.6, and $3.9 \%$, respectively, for girls (Fig. 2). Boys showed a distinct decrease in the number of steps per day between the sixth primary school grade and the first junior high school grade. Girls showed a gradual decline in the number of steps per day from the start to the finish of primary school, with a slight increase during the first year of junior high school.

The proportion of students taking $<7000$ and $<5000$ steps per day was 14.1 and $4.7 \%$, respectively, for boys and 20.7 and $5.0 \%$, respectively, for girls (Fig. 3). The proportion of boys taking $<7000$ and $<5000$ steps per day rapidly increased between the sixth grade of primary school and the first grade of junior high school, and continued to increase toward high school. The proportion of girls taking $<7000$ steps per day gradually increased from the first grade level to the last year of junior high school. There was a moderate relative decrease in the proportion of girls taking $<7000$ steps per day in the first year of high school, but a subsequent steady increase in later years.

\section{Discussion}

Using a representative sample of the Tokyo metropolitan area, this is one of the largest surveys worldwide to investigate pedometer-determined PA levels in children and adolescents. The results indicate that in primary school (age 6-12 years), junior high (age 12-15 years), and high school (age 15-18 years), boys took an average of 12,483, 9476, and 8294 steps per day, respectively, while girls took an average of 10,053, 8408, and 8184 steps per day, respectively. The mean number of daily steps was significantly higher for boys than for girls through 6 to 15 years, with an overall decreasing agerelated trend for both sexes. Boys tend to be more active than girls at most ages, although this difference disappears in high school, and a reduction in PA levels from childhood to adolescence has been previously reported $[17,18,25]$. Until now, there has been limited objective data for large-scale evaluations of PA levels in Japanese children and adolescents [5]. The step patterns of our Tokyo students are similar to those reported in a review of pedometer data from 43 studies of young people in 13 countries [30].

Although high school is not compulsory in Japan, more than $95 \%$ of students in Tokyo attend high school after passing their entrance examination [19]. Generally, Japanese students in the third year of junior high school spend substantial time studying for this examination. Therefore, students in their final junior high school year may focus more on studying than on PA, which may explain why this group has the lowest overall mean step count and the highest proportion of youth accumulating fewer steps per day relative to the two indices of a sedentary lifestyle.

The differences in PA levels between children in Tokyo and those in Canada can be interpreted in four ways. First, Canadian and Japanese children may actually have different PA levels. Second, this difference may result from variations in the survey-specific pedometers used and their positioning. The EX-200 (used in this survey) is an in-pocket pedometer and the SW-200 (used in the CANPLAY survey) is worn on a belt. The EX-200 is a triaxial accelerometer with a filter function that monitors continuous walking activity to recognize actual steps; it is programmed to count steps when an individual takes $\geq 10$ steps without pausing for $<2 \mathrm{~s}$ (e.g., if a subject moves $<10$ steps and pauses for $\geq 2 \mathrm{~s}$, the previous steps will not be counted). Silcott et al. [31] reported that pedometers with a filter function might underestimate step counts compared with pedometers without a filter function. Although there is no data directly comparing steps measured by the EX200 and the SW-200, Tanaka et al. reported that the EX-200 underestimated step counts by $7.9 \%$ compared with the Kenz Lifecorder among children aged 6 to 12 years [22] (additionally, Schneider et al. reported no significant difference in step count values between the Kenz Lifecorder and the SW200 [21]). These findings suggest that the EX-200 step counts may be lower than those obtained by the SW-200. Thus, differences among devices should be considered. Third, the sampling method in this TMBE-administered survey was different from the CANPLAY which employed random sampling and collected data through the mail. In 
Table 3 Normative steps per day by grade level and sex in Tokyo in 2011

\begin{tabular}{|c|c|c|c|c|c|c|c|c|c|c|c|c|c|c|}
\hline & \multirow[b]{2}{*}{ Grade } & \multirow[b]{2}{*}{ Age } & \multirow[b]{2}{*}{ Minimum } & \multicolumn{11}{|c|}{ Percentiles } \\
\hline & & & & 1 & 3 & 5 & 10 & 15 & 20 & 25 & 30 & 35 & 40 & 45 \\
\hline \multicolumn{15}{|l|}{ Boys } \\
\hline \multirow[t]{6}{*}{ Primary school } & 1 & $6-7$ & 1523 & 2767 & 6569 & 7563 & 8743 & 9310 & 9996 & 10423 & 10742 & 11259 & 11663 & 12010 \\
\hline & 2 & $7-8$ & 1005 & 4789 & 6582 & 7264 & 8509 & 9210 & 9822 & 10314 & 10822 & 11205 & 11732 & 12268 \\
\hline & 3 & $8-9$ & 1032 & 4639 & 6148 & 7183 & 8377 & 9467 & 9939 & 10345 & 10880 & 11293 & 11763 & 12128 \\
\hline & 4 & $9-10$ & 1591 & 4343 & 6105 & 6971 & 7937 & 8929 & 9710 & 10260 & 10680 & 11027 & 11579 & 12087 \\
\hline & 5 & $10-11$ & 1987 & 4340 & 6136 & 7091 & 8019 & 8774 & 9345 & 9760 & 10236 & 10613 & 11229 & 11556 \\
\hline & 6 & $11-12$ & 1846 & 3967 & 5609 & 6321 & 7298 & 8309 & 8910 & 9546 & 9991 & 10508 & 10908 & 11389 \\
\hline \multirow[t]{3}{*}{ Junior high school } & 1 & $12-13$ & 1035 & 2535 & 4290 & 4785 & 5892 & 6454 & 7104 & 7591 & 7929 & 8294 & 8731 & 9122 \\
\hline & 2 & $13-14$ & 1034 & 2544 & 3822 & 4252 & 5304 & 6169 & 6644 & 7120 & 7524 & 8013 & 8362 & 8735 \\
\hline & 3 & $14-15$ & 1000 & 2096 & 3324 & 3882 & 4800 & 5283 & 5789 & 6069 & 6539 & 6870 & 7135 & 7477 \\
\hline \multirow[t]{3}{*}{ High school } & 1 & $15-16$ & 2171 & 3235 & 3922 & 4214 & 4719 & 5298 & 5592 & 6212 & 6560 & 6951 & 7268 & 7488 \\
\hline & 2 & $16-17$ & 1410 & 2108 & 3016 & 3288 & 4185 & 4606 & 5246 & 5714 & 6079 & 6695 & 6988 & 7408 \\
\hline & 3 & $17-18$ & 1007 & 1146 & 2922 & 3317 & 3914 & 4372 & 5033 & 5343 & 6118 & 6393 & 6733 & 7081 \\
\hline \multicolumn{15}{|l|}{ Girls } \\
\hline \multirow[t]{6}{*}{ Primary school } & 1 & $6-7$ & 1269 & 3846 & 6201 & 6807 & 7668 & 8172 & 8621 & 8939 & 9262 & 9647 & 10072 & 10424 \\
\hline & 2 & $7-8$ & 1511 & 3987 & 5933 & 6563 & 7352 & 7806 & 8346 & 8737 & 9128 & 9415 & 9767 & 10114 \\
\hline & 3 & $8-9$ & 1315 & 4018 & 5698 & 6197 & 7224 & 7818 & 8269 & 8668 & 9028 & 9327 & 9645 & 9935 \\
\hline & 4 & $9-10$ & 1549 & 4121 & 5544 & 6131 & 6789 & 7297 & 7877 & 8190 & 8551 & 8960 & 9315 & 9716 \\
\hline & 5 & $10-11$ & 2697 & 4024 & 5110 & 5637 & 6461 & 7020 & 7508 & 7878 & 8163 & 8492 & 8732 & 9099 \\
\hline & 6 & $11-12$ & 1953 & 3953 & 5171 & 5590 & 6400 & 6791 & 7067 & 7487 & 7753 & 8033 & 8252 & 8622 \\
\hline \multirow[t]{3}{*}{ Junior high school } & 1 & $12-13$ & 1833 & 2880 & 3777 & 4445 & 5231 & 5758 & 6296 & 6649 & 7001 & 7386 & 7766 & 8265 \\
\hline & 2 & $13-14$ & 1451 & 3176 & 4042 & 4466 & 5298 & 5786 & 6231 & 6572 & 6909 & 7211 & 7521 & 7873 \\
\hline & 3 & $14-15$ & 1346 & 2705 & 3640 & 4213 & 4711 & 5129 & 5447 & 5757 & 6134 & 6374 & 6669 & 6862 \\
\hline \multirow[t]{3}{*}{ High school } & 1 & $15-16$ & 1086 & 2037 & 3362 & 3797 & 4722 & 5103 & 5828 & 6369 & 7027 & 7277 & 7530 & 7798 \\
\hline & 2 & $16-17$ & 3126 & 3227 & 3814 & 4396 & 5091 & 5191 & 5628 & 6108 & 6454 & 6903 & 7142 & 7388 \\
\hline & 3 & $17-18$ & 3000 & 3115 & 3646 & 4518 & 4966 & 5565 & 6135 & 6292 & 6684 & 6942 & 7158 & 7462 \\
\hline
\end{tabular}


Table 3 Normative steps per day by grade level and sex in Tokyo in 2011 (Continued)

\begin{tabular}{|c|c|c|c|c|c|c|c|c|c|c|c|c|c|}
\hline & \multicolumn{12}{|c|}{ Percentiles } & \multirow[b]{2}{*}{ Maximum } \\
\hline & 50 & 55 & 60 & 65 & 70 & 75 & 80 & 85 & 90 & 95 & 97 & 99 & \\
\hline \multicolumn{14}{|l|}{ Boys } \\
\hline \multirow[t]{6}{*}{ Primary school } & 12397 & 12905 & 13292 & 13776 & 14146 & 14794 & 15268 & 16021 & 16813 & 18004 & 18886 & 22157 & 24315 \\
\hline & 12660 & 13108 & 13572 & 14002 & 14336 & 14860 & 15408 & 16305 & 17101 & 18630 & 19725 & 22100 & 26775 \\
\hline & 12563 & 13059 & 13483 & 13983 & 14496 & 14962 & 15522 & 16264 & 17187 & 18627 & 19893 & 21601 & 29999 \\
\hline & 12536 & 13027 & 13491 & 13927 & 14506 & 14952 & 15606 & 16284 & 17344 & 18436 & 19545 & 21291 & 27000 \\
\hline & 11992 & 12460 & 12864 & 13391 & 13885 & 14478 & 15087 & 16174 & 17083 & 18567 & 19522 & 21678 & 29994 \\
\hline & 11726 & 12168 & 12794 & 13248 & 13825 & 14355 & 14977 & 15675 & 16684 & 18540 & 19457 & 21787 & 30000 \\
\hline \multirow[t]{3}{*}{ Junior high school } & 9516 & 9982 & 10605 & 11198 & 11811 & 12441 & 13186 & 14250 & 15430 & 17732 & 18775 & 21286 & 29265 \\
\hline & 9224 & 9645 & 10242 & 10755 & 11472 & 12173 & 12832 & 13727 & 15003 & 17200 & 18263 & 21536 & 27659 \\
\hline & 7802 & 8160 & 8642 & 8994 & 9428 & 9973 & 10441 & 11249 & 12381 & 14799 & 16217 & 19984 & 26603 \\
\hline \multirow[t]{3}{*}{ High school } & 7951 & 8187 & 8578 & 9009 & 9516 & 10012 & 10641 & 11556 & 13316 & 16905 & 18602 & 21309 & 24046 \\
\hline & 7760 & 8158 & 8560 & 8764 & 8984 & 9662 & 10952 & 12146 & 14043 & 16059 & 17676 & 20211 & 20311 \\
\hline & 7439 & 7758 & 8519 & 9114 & 9354 & 10007 & 10375 & 11354 & 12366 & 14254 & 15746 & 18292 & 19257 \\
\hline \multicolumn{14}{|l|}{ Girls } \\
\hline \multirow[t]{6}{*}{ Primary school } & 10715 & 10972 & 11263 & 11585 & 11886 & 12293 & 12746 & 13251 & 13837 & 14779 & 15708 & 17307 & 21908 \\
\hline & 10441 & 10766 & 11148 & 11492 & 11824 & 12318 & 12712 & 13295 & 13966 & 15249 & 16348 & 17919 & 20879 \\
\hline & 10219 & 10639 & 11025 & 11299 & 11625 & 12095 & 12557 & 13323 & 14019 & 14954 & 15796 & 17877 & 24133 \\
\hline & 9952 & 10176 & 10450 & 10772 & 11101 & 11469 & 11943 & 12482 & 13186 & 14596 & 15476 & 17789 & 22450 \\
\hline & 9372 & 9698 & 10019 & 10317 & 10669 & 10977 & 11415 & 11887 & 12777 & 13745 & 14944 & 15826 & 22195 \\
\hline & 8884 & 9234 & 9532 & 9832 & 10103 & 10427 & 10902 & 11399 & 12153 & 13626 & 14504 & 16596 & 20504 \\
\hline \multirow[t]{3}{*}{ Junior high school } & 8648 & 8969 & 9410 & 9879 & 10426 & 11077 & 11856 & 12760 & 14001 & 15518 & 16216 & 18181 & 23343 \\
\hline & 8212 & 8542 & 8820 & 9273 & 9732 & 10151 & 10791 & 11519 & 12893 & 15101 & 16404 & 18749 & 24388 \\
\hline & 7144 & 7386 & 7699 & 8089 & 8408 & 8707 & 9140 & 9806 & 10474 & 11687 & 12625 & 14656 & 20730 \\
\hline \multirow[t]{3}{*}{ High school } & 8141 & 8403 & 8955 & 9329 & 9689 & 9971 & 10623 & 11447 & 12799 & 14323 & 14889 & 16004 & 17258 \\
\hline & 7656 & 8010 & 8351 & 8663 & 9024 & 9320 & 9919 & 10570 & 11422 & 12787 & 14899 & 16251 & 20525 \\
\hline & 7592 & 7864 & 8320 & 8697 & 9305 & 9694 & 9989 & 10531 & 11570 & 14000 & 14864 & 16570 & 17308 \\
\hline
\end{tabular}


Table 4 Comparison of mean number of steps per day among boys and girls between Canada (in 2005-2011) and Tokyo in 2011

\begin{tabular}{|c|c|c|c|c|}
\hline \multirow[b]{3}{*}{ Pedometer } & \multicolumn{2}{|l|}{ Boys } & \multicolumn{2}{|l|}{ Girls } \\
\hline & Canada $^{a}$ & Tokyo & Canada $^{a}$ & Tokyo \\
\hline & Yamax SW-200 & Yamasa Ex-200 & Yamax SW-200 & Yamasa Ex-200 \\
\hline Age & Steps per day & Steps per day & Steps per day & Steps per day \\
\hline 6 & 12,435 & 12,575 & 11,627 & 10,694 \\
\hline 7 & 12,700 & 12,714 & 11,507 & 10,573 \\
\hline 8 & 12,989 & 12,736 & 11,435 & 10,452 \\
\hline 9 & 13,097 & 12,596 & 11,490 & 10,010 \\
\hline 10 & 13,030 & 12,302 & 11,638 & 9517 \\
\hline 11 & 12,694 & 12,021 & 11,367 & 9117 \\
\hline 12 & 12,211 & 10,218 & 10,510 & 9104 \\
\hline 13 & 11,816 & 9830 & 10,122 & 8689 \\
\hline 14 & 11,114 & 8337 & 9988 & 7437 \\
\hline 15 & 10,650 & 8583 & 9476 & 8398 \\
\hline 16 & 10,344 & 8322 & 9252 & 8025 \\
\hline 17 & 10,493 & 7935 & 9343 & 8130 \\
\hline
\end{tabular}

${ }^{a}$ The Canadian Physical Activity Levels Among Youth (CANPLAY) survey [16]. Yamax SW-200 pedometers were used in the CANPLAY survey, and Yamasa EX-200 pedometers in the Tokyo survey. The SW-200 was worn on a belt, the EX-200 was placed in a pocket

contrast, the Tokyo survey asked each municipality to choose one primary and one junior high school from its district. All geographical areas throughout Tokyo were covered by this method. However, it is uncertain whether this sampling method lead to underestimation or overestimation of the step counts. Finally, children's activity levels may be affected by seasonal variation [32]. Craig et al. [15] reported that Canadian children's PA is lower in the fall and winter than in the spring and summer. Because this survey was conducted in the fall in Tokyo, further study is needed to determine the effect of seasonal changes on PA levels in Japanese children and adolescents.

Vincent et al. [33] assessed pedometer-determined daily step counts in a convenience sample of children aged 6 to 12 years in the U.S. $(n=711)$, Sweden $(n=680)$, and Australia $(n=563)$. Although there are potential issues with the sample size, sampling bias, and the different pedometers used in the present survey, the Tokyo survey reported approximately 3000 and 1000 fewer steps for boys per day than Swedish and Australian boys, respectively, but a similar level of activity to American boys. We observed a similar pattern for girls, who took approximately 2000 and 1000 fewer steps per day than Swedish and Australian girls, respectively, but accumulated a similar mean number of steps per day as American girls.

There are no clear guideline recommendations for number of steps per day for children and adolescents. However, we interpreted the present data in the context of previously published step-defined criteria. Certainly, it is optimal to use criteria that have been established based on health-related values. However, practical effectiveness should also be taken into consideration when setting any single criterion. For example, if almost all (or very few) people meet a specified value, then its practical effectiveness for educational and public health purposes is questionable. The present results include implications regarding the practical effectiveness of various criteria. We found that the proportion of boys and girls meeting specific criteria (i.e., 10,000, 12,000, and 15,000 steps per day) decreased with age. Additionally, distinct sex-specific patterns were observed. The Tokyo Metropolitan Government has recommended $\geq 15,000$ steps per day for children and adolescents, regardless of age or sex [27]. However, our findings reveal that many children and adolescents (except primary school boys) do not meet this target. Therefore, a criterion of $\geq 15,000$ steps per day seems very high and thus not practically effective, especially for girls. Two courses of action might improve the situation. First, appropriate age- and sex-specific targets may be set. Second, a graduated scale of values might describe PA distribution better than a single target value and may encourage less active children to improve their PA level. Although it seems too low as an optimal value for health, a criterion of $\geq 10,000$ steps per day showed dynamic patterns across age and sex in this survey. This suggests that $\geq 10,000$ steps per day is a practically effective criterion for evaluating lifestyle changes/differences across age and sex for education and public health purposes. For children, $<7000$ steps per day has been suggested as an appropriate sedentary lifestyle index $[15,28]$. In this survey, the proportion of students meeting this criterion increased with age as anticipated. However, 


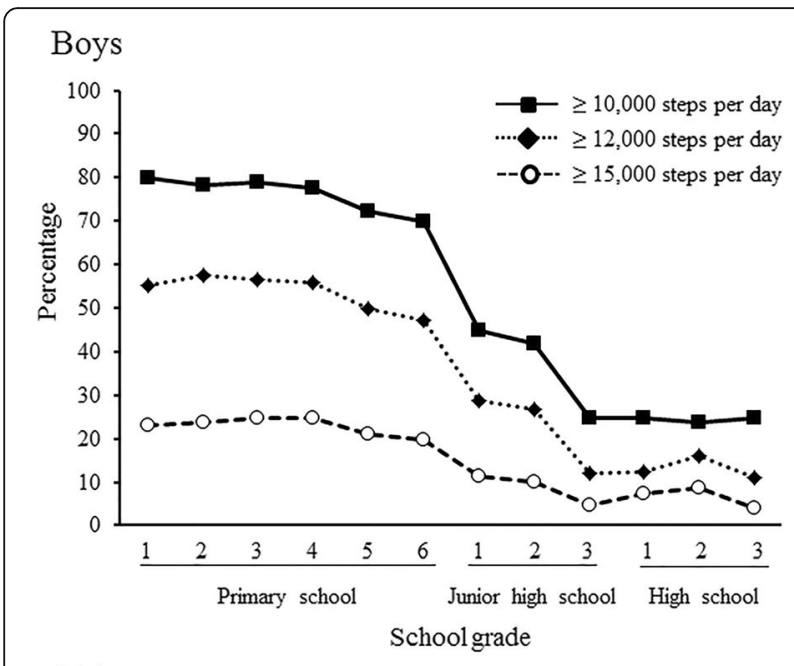

Girls

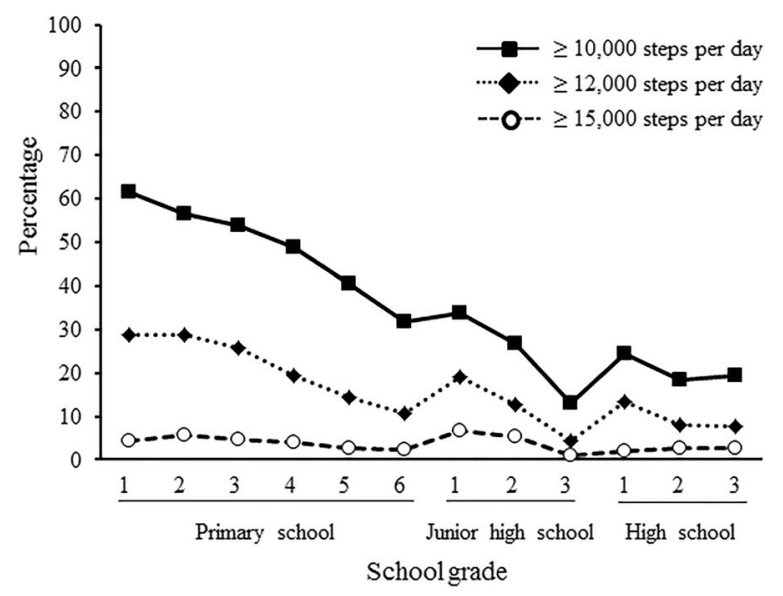

Fig. 2 Proportion of students taking $\geq 10,000, \geq 12,000$, or $\geq 15,000$ steps per day by sex and school grade

its practical effectiveness was limited for primary school boys, who accumulated a higher average number of steps per day than this value, suggesting the need for age- and sex-specific values on a graduated scale for youth.

In the present study, the use of unsealed pedometers meant that participants were aware of their step counts, potentially leading to reactivity bias. Because TMBE did not record the steps per day for the first 7 days, our ability to test for reactivity was hampered. However, Craig et al. [23] showed no evidence of reactivity in a population sample of 5- to 19-year-olds wearing unsealed pedometers for 7 days. Other studies have reported no evidence of reactivity bias and have generally concluded that this is not a problem when evaluating children [13]. Additionally, Clemes and Deans [34] reported that the reactivity effect diminishes after the first week of monitoring, returning to normal levels in the second week. Therefore, the data

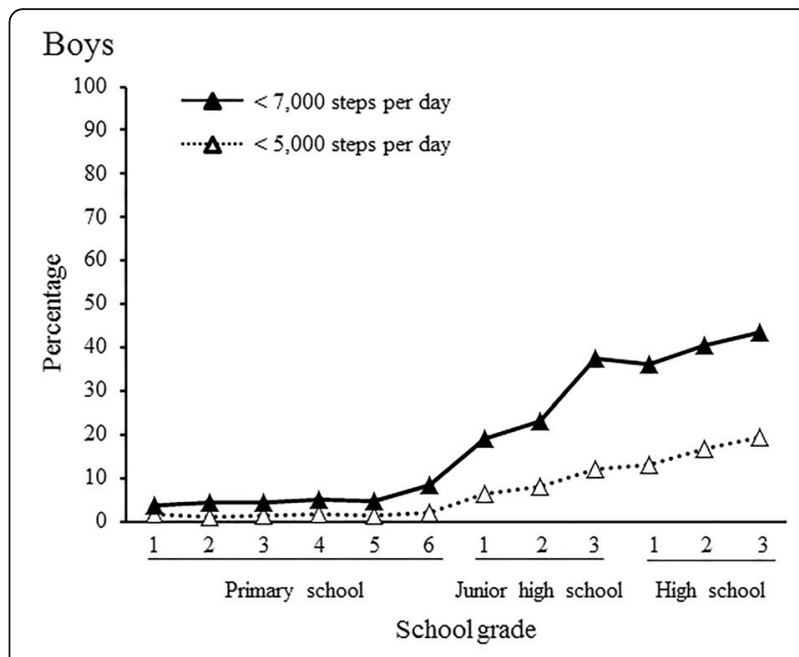

Girls

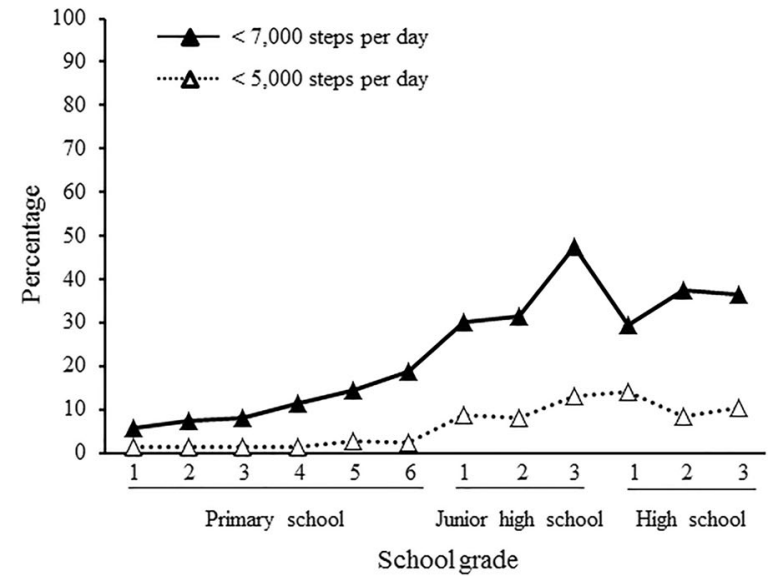

Fig. 3 Proportion of students taking $<7,000$ or $<5,000$ steps per day by sex and school grade

obtained in the second week of our 2-week surveillance were probably not systematically affected by reactivity bias.

\section{Study strengths and limitations}

This study has several strengths. Because the participants were sampled throughout Tokyo, their mean number of steps per day is representative of PA levels in Tokyo youth. Using the same adjusted treatment methods for pedometer data as used in the CANPLAY study enabled between-study comparisons. Finally, in this study, more than 86 and $90 \%$ of boys and girls, respectively, wore their pedometers for a minimum of 4 days; it has been reported that 4 or more valid days of data in youth enhances data reliability [35].

Study limitations must be acknowledged. First, this was that was a secondary analysis of a survey conducted by an education authority and we had no input regarding the original study design. Despite this, the survey represents an important source of objectively monitored 
data on children. Although this is the largest study of inpocket pedometer-determined PA in youth (and is thus a useful reference data source for others using this type of device), these pedometers do tend to underestimate absolute step-defined PA levels. Regardless, it is reasonable to assume that the observed data trends are valid. Second, the lack of private school students in the sample may influence the results. In 2011, the proportion of students in the Tokyo metropolitan area attending private primary, junior high, and high schools was 4.5, 25.5, and $55.9 \%$, respectively [36]. The difference in tuition costs for private schools may indicate differences in familial socioeconomic status. If socioeconomic status affects youth PA levels, the present data may not accurately reflect PA in the larger Tokyo youth population. Third, the TMBE survey complied with the organization's safety policy, thus allowing students to remove their pedometers during vigorous fullcontact activity (unfortunately, this was not tracked); this may have underestimated the overall number of steps per day. Finally, the issue of wearing compliance should be considered. Students recorded their step counts at school under the guidance of trained teachers. However, no other methods were employed to confirm whether they actually wore the pedometers as directed.

\section{Conclusion}

This study demonstrates that children's pedometerdetermined PA generally decreases with age and that there is a substantial difference in the number of steps taken per day between boys and girls in Tokyo. The PA decrease was greater in boys because they achieved initial higher peak values; once the students reached high school, the sex difference in the number of steps per day disappeared. These findings contribute to our current understanding of the PA levels of youth living in Tokyo and will be useful for surveillance, screening, and comparison purposes, as well as planning strategies.

\section{Abbreviations}

CANPLAY: Canadian physical activity levels among youth; Cl: Confidence interval; PA: Physical activity; TMBE: Tokyo Metropolitan board of education

\section{Acknowledgements}

We thank all the study participants and data collectors for their willingness to participate in this survey. We also thank all members of the Tokyo Metropolitan Board of Education and the Tokyo Metropolitan Government who helped make this study possible.

\section{Availability of data and materials}

The data described herein come from a secondary use of dataset collected by the Tokyo Metropolitan Board of Education with their permission. The data is not permitted to be opened for public use.

\section{Authors' contributions}

SI conceived the study. NF carried out the analysis, and drafted the manuscript. SI, CTL, and ST interpreted results and critically edited the manuscript. HS and HK provided statistical expertise, and contributed to discussion. YH advised on and reviewed data analysis, and contributed to discussion. All authors read and approved the final manuscript.

\section{Competing interests}

The author declares that they have no competing interests.

Consent for publication

Not applicable.

Ethics approval and consent to participate

Ethical approval was obtained from the Tokyo Medical University Ethics Committee (No. 2762). Data were collected as administrative data by the Tokyo Metropolitan Board of Education and the Tokyo Metropolitan Government. The Tokyo Metropolitan Board of Education and the Tokyo Metropolitan Government approved the secondary use of these data for research purposes.

\section{Author details}

${ }^{1}$ Department of Preventive Medicine and Public Health, Tokyo Medical University, 6-1-1 Shinjuku Shinjuku-ku, Tokyo 160-8402, Japan. Faculty of Engineering, Chiba Institute of Technology, 2-1-1 Shibazono, Narashino, Chiba 275-0023, Japan. ${ }^{3}$ Department of Kinesiology, School of Public Health and Health Sciences, University of Massachusetts Amherst, 111 Totman Building University of Massachusetts Amherst 30 Eastman Lane, Amherst, MA 01003-9258, USA. ${ }^{4}$ Department of Nutritional Science, National Institute of Health and Nutrition, National Institutes of Biomedical Innovation, Health and Nutrition, 1-23-1 Toyama, Shinjuku-ku, 162-8636 Tokyo, Japan.

Received: 8 July 2016 Accepted: 15 October 2016

Published online: 21 October 2016

\section{References}

1. Ekelund U, Brage S, Froberg K, Harro M, Anderssen SA, Sardinha LB, Riddoch C, Andersen LB. TV viewing and physical activity are independently associated with metabolic risk in children: the European youth heart study. PLoS Med. 2006;3(12):e488.

2. Janssen I, Katzmarzyk PT, Boyce WF, Vereecken C, Mulvihill C, Roberts C, Currie C, Pickett W. Comparison of overweight and obesity prevalence in school-aged youth from 34 countries and their relationships with physical activity and dietary patterns. Obes Rev. 2005;6(2):123-32.

3. Trudeau F, Laurencelle L, Shephard RJ. Tracking of physical activity from childhood to adulthood. Med Sci Sports Exerc. 2004;36(11):1937-43.

4. World Health Organization. Geneva (CH): Global Strategy on Diet, Physical Activity and Health. http://www.who.int/dietphysicalactivity/factsheet_ young_people/en/ Accessed 4 Oct 2016.

5. Hallal PC, Andersen LB, Bull FC, Guthold R, Haskell W, Ekelund U. Global physical activity levels: surveillance progress, pitfalls, and prospects. Lancet. 2012;380(9838):247-57.

6. Sisson SB, Katzmarzyk PT. International prevalence of physical activity in youth and adults. Obes Rev. 2008:9(6):606-14

7. Sallis JF. Self-report measures of children's physical activity. J Sch Health. 1991;61(5):215-9.

8. Bailey RC, Olson J, Pepper SL, Porszasz J, Barstow TJ, Cooper DM. The level and tempo of children's physical activities: an observational study. Med Sc Sports Exerc. 1995;27:1033-41.13.

9. Welk GJ, Corbin CB, Dale D. Measurement issues in the assessment of physical activity in children. Res Q Exerc Sport. 2000;71 Suppl 2:S59-73.

10. Johnson TP, Cho YI, Holbrook AL, O'Rourke D, Warnecke RB, Chavez N. Cultural variability in the effects of question design features on respondent comprehension of health surveys. Ann Epidemiol. 2006;16(9):661-8.

11. Sallis JF, Saelens BE. Assessment of physical activity by self-report: status, limitations, and future directions. Res Q Exerc Sport. 2000;71 Suppl 2:S1-14.

12. Warnecke RB, Johnson TP, Chavez N, et al. Improving question wording in surveys of culturally diverse populations. Ann Epidemiol. 1997;7(5):334-42.

13. Clemes SA, Biddle SJ. The use of pedometers for monitoring physical activity in children and adolescents: measurement considerations. J Phys Act Health. 2013;10:249-62.

14. De Vries SI, Van Hirtum HW, Bakker I, Hopman-Rock M, Hirasing RA, Van Mechelen W. Validity and reproducibility of motion sensors in youth: a systematic update. Med Sci Sports Exerc. 2009;41(4):818-27.

15. Craig CL, Cameron C, Griffiths JM, Tudor-Locke C. Descriptive epidemiology of youth pedometer-determined physical activity: CANPLAY. Med Sci Sports Exerc. 2010;42:1639-43. 
16. Craig CL, Cameron C, Tudor-Locke C. CANPLAY pedometer normative reference data for 21,271 children and 12,956 adolescents. Med Sci Sports Exerc. 2013;45(1):123-9.

17. Tudor-Locke C, Johnson WD, Katzmarzyk PT. Accelerometer-determined steps per day in US children and youth. Med Sci Sports Exerc. 2010;42(12):2244-50

18. Riddoch CJ, Bo Andersen L, Wedderkopp N, Harro M, Klasson-Heggebo L, Sardinha LB, Cooper AR, Ekelund U. Physical activity levels and patterns of 9- and 15-yr-old European children. Med Sci Sports Exerc. 2004;36(1):86-92.

19. Tokyo Metropolitan Board of Education Web site. Tokyo (JP): Tokyo Metropolitan Board of Education; http://www.kyoiku.metro.tokyo.jp/toukei/ 23sotsugo/toppage.htm. Accessed 4 Oct 2016

20. Rowe DA, Mahar MT, Raedeke TD, et al. Measuring physical activity in children with pedometers: reliability, reactivity, and replacement of missing data. Pediatr Exerc Sci. 2004;16:343-54.

21. Schneider PL, Crouter S, Bassett DR. Pedometer measures of free-living physical activity: comparison of 13 models. Med Sci Sports Exerc. 2004;36:331-5.

22. Tanaka S, Inoue S, Hikihara Y, Tanaka C. Development of conversion equations for adjustment of discrepancy of step counts between pedometers. Res Papers Suzuken Memorial Foundation. 2012;31:96-9. Japanese.

23. Craig CL, Tudor-Locke C, Cragg S, Cameron C. Process and treatment of pedometer data collection for youth: the Canadian physical activity levels among Youth study. Med Sci Sports Exerc. 2010;42(3):430-5.

24. Tudor-Locke C, Johnson WD, Katzmarzyk PT. Accelerometer-determined steps per day in US adults. Med Sci Sports Exerc. 2009;41(7):1384-91.

25. Tudor-Locke C, Craig CL, Beets MW, Belton S, Cardon GM, Duncan S, Hatano Y, Lubans DR, Olds TS, Raustorp A, Rowe DA, Spence JC, Tanaka S, Blair SN. How many steps/day are enough? for children and adolescents. Int J Behav Nutr Phys Act. 2011;8:78.

26. Tudor-Locke C, Pangrazi RP, Corbin CB, Rutherford WJ, Vincent SD, Raustorp A, Tomson LM, Cuddihy TF. BMI-referenced standards for recommended pedometer-determined steps/day in children. Prev Med. 2004;38(6):857-64.

27. Tokyo Metropolitan Board of Education Web site [Internet]. Tokyo (JP): Tokyo Metropolitan Board of Education; http://www.kyoiku.metro.tokyo.jp/ pickup/seisaku_sport.htm. Accessed 4 Oct 2016.

28. Tudor-Locke C, Craig CL, Thyfault JP, Spence JC. A step-defined sedentary lifestyle index: <5000 steps/day. Appl Physiol Nutr Metab. 2013;38:100-14.

29. Cohen J. A power primer. Psychol Bull. 1992;112:155-9.

30. Beets MW, Bornstein D, Beighle A, Cardinal BJ, Morgan CF. Pedometermeasured physical activity patterns of youth: a 13-country review. Am J Prev Med. 2010;38:208-16.

31. Silcott NA, Bassett Jr DR, Thompson DL, Fitzhugh EC, Steeves JA. Evaluation of the Omron HJ-720ITC pedometer under free-living conditions. Med Sci Sports Exerc. 2011:43:1791-7.

32. Rich C, Griffiths LJ, Dezateux C. Seasonal variation in accelerometerdetermined sedentary behaviour and physical activity in children: a review. Int J Behav Nutr Phys Act. 2012;9:49.

33. Vincent SD, Pangrazi RP, Raustorp A, Tomson LM, Cuddihy TF. Activity levels and body mass index of children in the United States, Sweden, and Australia. Med Sci Sports Exerc. 2003;35(8):1367-73.

34. Clemes SA, Deans NK. Presence and duration of reactivity to pedometers in adults. Med Sci Sports Exerc. 2012;44:1097-101.

35. Corder K, Ekelund U, Steele RM, Wareham NJ, Brage S. Assessment of physical activity in youth. J Appl Physiol. 2008;105:977-87.

36. Tokyo Metropolitan Government Web site [Internet]. Tokyo (JP): The School Basic Survey 2011; http://www.toukei.metro.tokyo.jp/gakkou/2011/ gk11gg10000.htm. Accessed 4 Oct 2016.

\section{Submit your next manuscript to BioMed Central and we will help you at every step:}

- We accept pre-submission inquiries

- Our selector tool helps you to find the most relevant journal

- We provide round the clock customer support

- Convenient online submission

- Thorough peer review

- Inclusion in PubMed and all major indexing services

- Maximum visibility for your research

Submit your manuscript at www.biomedcentral.com/submit
Biomed Central 